\title{
On uniqueness and stability for supercritical nonlinear wave and Schrödinger equations
}

\section{Journal Article}

Author(s):

Struwe, Michael

Publication date:

2006

Permanent link:

https://doi.org/10.3929/ethz-b-000036098

Rights / license:

In Copyright - Non-Commercial Use Permitted

Originally published in:

International Mathematics Research Notices 2006, https://doi.org/10.1155/IMRN/2006/76737 


\title{
On Uniqueness and Stability for Supercritical Nonlinear Wave and Schrödinger Equations
}

\author{
Michael Struwe
}

\section{Introduction}

In a previous paper [10], it was shown how uniqueness of classical solutions to critical nonlinear wave equations like

$$
u_{t t}-\Delta u+u^{5}=0 \quad \text { on } \mathbb{R} \times \mathbb{R}^{3}
$$

with data

$$
\left(u, u_{t}\right)_{\left.\right|_{t=0}}=\left(u_{0}, u_{1}\right) \in C_{0}^{\infty} \times C_{0}^{\infty}\left(\mathbb{R}^{3}\right)
$$

can be obtained in the a priori much larger class of distribution solutions satisfying the energy inequality. At a conference at the Bernoulli Center of EPFL Lausanne in June 2004, I pointed out that the method can be applied without any changes also in the supercritical case when the nonlinearity $f(u)=u^{5}$ is substituted by the function $f(u)=$ $u^{2 k+1}$ for any $k \in \mathbb{N}$, in any number of space dimensions. In fact, as we show here, the same method also works for more general nonlinearities and in the case of nonlinear Schrödinger equations. 
Michael Struwe

\section{Nonlinear wave equations}

Consider the Cauchy problem for the equation

$$
u_{t t}-\Delta u+m u+f(u)=0 \quad \text { on } \mathbb{R} \times \mathbb{R}^{n}
$$

with data

$$
\left(u, u_{t}\right)_{\left.\right|_{t=0}}=\left(u_{0}, u_{1}\right) \in C_{0}^{\infty} \times C_{0}^{\infty}\left(\mathbb{R}^{n}\right)
$$

where $m \geq 0$ and where $f=F^{\prime}$ for some $C^{2}$-function $F: \mathbb{R} \rightarrow \mathbb{R}$ satisfying

$$
0 \leq u f(u) \leq C F(u)
$$

with some uniform constant $C$. Moreover, we request polynomial-type behavior in the sense that for all $R>0$ there exist numbers $\varepsilon=\varepsilon(R)>0, C=C(R)$ such that the conditions

$$
\mathrm{F}(\mathrm{u}+w)-\mathrm{F}(\mathrm{u})-\mathrm{f}(\mathrm{u}) w \geq \varepsilon \mathrm{F}(w)-\mathrm{C}|w|^{2}
$$

as well as

$$
\begin{gathered}
F(u+w)-F(u)-f(u) w \leq C F(w)+C|w|^{2}, \\
\left|f(u+w)-f(u)-f^{\prime}(u) w\right| \leq C F(w)+C|w|^{2}
\end{gathered}
$$

hold true for all $w$ whenever $|u| \leq R$. Clearly, we may assume that $\varepsilon \leq 1$. The conditions (2.3)-(2.6) are satisfied for $F(u)=|u|^{p}$ for any $p \geq 2$; however, they fail to hold, for example, when $\mathrm{F}(\mathrm{u})=\mathrm{e}^{\mathrm{u}^{2}}-1$.

For classical solutions $u$ of (2.1), upon multiplying (2.1) by $u_{t}$ and integrating over any time-slice $[0, t] \times \mathbb{R}^{n}$, one easily finds the energy identity

$$
E(u(t))=\int_{\{t\} \times \mathbb{R}^{n}}\left(\frac{|D u|^{2}+m u^{2}}{2}+F(u)\right) d x=E(u(0)),
$$

where $D u=\left(u_{t}, \nabla u\right)$ is the space-time gradient. Moreover, these solutions have spatially compact support and therefore together with their derivatives are uniformly bounded on any such time-slice.

In the case of nonlinearities with "supercritical" growth, the Cauchy problem (2.1), (2.2) is not known to admit global smooth solutions. However, by results of Segal [6], Lions [5], and Strauss [8], assuming (2.3) we always can obtain weak solutions 
to this equation with $\mathrm{Du}$, $m u \in \mathrm{L}^{\infty}\left(\mathbb{R} ; \mathrm{L}^{2}\left(\mathbb{R}^{\mathfrak{n}}\right)\right)$ and with $\mathrm{F}(\mathrm{u}) \in \mathrm{L}^{\infty}\left(\mathbb{R} ; \mathrm{L}^{1}\left(\mathbb{R}^{\mathfrak{n}}\right)\right)$, satisfying (2.1) in the sense of distributions and such that the energy inequality

$$
E(u(t)) \leq E(u(0))
$$

holds for all $t$; see, for instance, [7, Chapter 6.2] or [9, Theorem 3.1]. We call such solutions "of energy class." Note that the map $t \mapsto D u(t) \in \mathrm{L}^{2}\left(\mathbb{R}^{n}\right)$ is weakly continuous for energyclass solutions $u$ of (2.1). The condition (2.8) and strict convexity of the $\mathrm{L}^{2}$-norm then imply that the initial data are continuously attained in the $\mathrm{H}^{1}$-norm.

We can now state the following uniqueness result.

Theorem 2.1. Suppose $u \in C^{\infty}\left(\mathbb{R} \times \mathbb{R}^{n}\right)$ is a classical solution to (2.1) with Cauchy data $\left(u, u_{t}\right)_{t=0}=\left(u_{0}, u_{1}\right) \in C_{0}^{\infty} \times C_{0}^{\infty}\left(\mathbb{R}^{n}\right)$, where $f$ satisfies (2.3)-(2.6). Also let $v$ be an energyclass solution to $(2.1),(2.2)$, satisfying $(2.8)$. Then $u \equiv v$.

Theorem 2.1 is a consequence of the following stability result.

Theorem 2.2. Suppose $u \in C^{\infty}\left(\mathbb{R} \times \mathbb{R}^{n}\right)$ is a classical solution to problem (2.1), (2.2), where $f$ satisfies (2.3)-(2.6). For $\left(v_{0}, v_{1}\right) \in \mathrm{H}^{1} \times \mathrm{L}^{2}\left(\mathbb{R}^{n}\right)$ with $\mathrm{F}\left(v_{0}\right) \in \mathrm{L}^{1}\left(\mathbb{R}^{n}\right)$, let $v$ be an energy-class solution to $(2.1)$ with Cauchy data $\left(v, v_{t}\right)_{\mid t=0}=\left(v_{0}, v_{1}\right)$ and satisfying the energy inequality

$$
\mathrm{E}(v(\mathrm{t})) \leq \mathrm{E}(v(0)) \quad \forall \mathrm{t}
$$

Then, letting $w=v-u$, if $m>0$ and if $u$ in addition is uniformly $C^{1}$-bounded in spacetime, for all $t \geq 0$ with constants $C_{i}=C_{i}(u)$, the following estimate holds:

$$
\mathrm{E}(w(\mathrm{t})) \leq \mathrm{C}_{1} e^{\mathrm{C}_{2} \mathrm{t}} \mathrm{E}(w(0)) .
$$

If $m=0$ or if $u$ fails to be uniformly bounded, for any time $T>0$ and any $0 \leq t \leq T$ with a constant $\mathrm{C}=\mathrm{C}(\mathrm{u}, \mathrm{T})$, there holds

$$
\mathrm{E}(w(\mathrm{t}))+\|w(\mathrm{t})\|_{\mathrm{L}^{2}}^{2} \leq \mathrm{C}\left(\mathrm{E}(w(0))+\|w(0)\|_{\mathrm{L}^{2}}^{2}\right) .
$$

Proof of Theorem 2.2. For ease of notation in the following, we consider only forward time $t \geq 0$. Observe that $w$ satisfies the equation

$$
w_{\mathrm{tt}}-\Delta w+m w+f(u+w)-f(u)=0 \quad \text { on } \mathbb{R} \times \mathbb{R}^{n}
$$


in the sense of distributions. Expand

$$
\frac{1}{2}|\mathrm{D} v|^{2}=\frac{1}{2}|\mathrm{Du}|^{2}+\mathrm{Du} \cdot \mathrm{D} w+\frac{1}{2}|\mathrm{D} w|^{2},
$$

and so on, so that

$$
E(v)=E(u)+I+I I,
$$

where

$$
\mathrm{I}=\langle\mathrm{dE}(\mathrm{u}), w\rangle=\int_{\mathbb{R}^{n}}(\mathrm{Du} \cdot \mathrm{D} w+\mathrm{muw}+\mathrm{f}(\mathrm{u}) w) \mathrm{dx}
$$

and with

$$
\mathrm{II}=\int_{\mathbb{R}^{n}}\left(\frac{|\mathrm{D} w|^{2}+m w^{2}}{2}+(\mathrm{F}(\mathrm{u}+w)-\mathrm{F}(\mathrm{u})-\mathrm{f}(\mathrm{u}) w)\right) \mathrm{d} x
$$

Choosing smooth functions $\eta_{k} \in C_{0}^{\infty}\left(\left[0, t[)\right.\right.$ with $0 \leq \eta_{k} \leq 1$ and converging almost everywhere to the characteristic function of the set $[0, t]$ as $k \rightarrow \infty$, we find

$$
I(t)-I(0)=\lim _{k \rightarrow \infty} \int_{0}^{t} \eta_{k} \frac{d}{d t}\langle d E(u), w\rangle d s
$$

where

$$
\begin{aligned}
\frac{d}{d t}\langle d E(u), w\rangle= & \int_{\mathbb{R}^{n}} \frac{d}{d t}(D u \cdot D w+m u w+f(u) w) d x \\
= & \int_{\mathbb{R}^{n}}\left(u_{t} w_{t}+\nabla u \cdot \nabla w_{t}+m u w_{t}+f(u) w_{t}\right) d x \\
& +\int_{\mathbb{R}^{n}}\left(u_{t} w_{t t}+\nabla u_{t} \cdot \nabla w+m u_{t} w+f^{\prime}(u) w u_{t}\right) d x
\end{aligned}
$$

may be interpreted in the distribution sense. Upon integrating by parts in the gradient terms and using (2.1) and (2.12), respectively, we then obtain

$$
\frac{d}{d t}\langle d E(u), w\rangle=\int_{\mathbb{R}^{n}}\left(f(u)+f^{\prime}(u) w-f(u+w)\right) u_{t} d x
$$

Using the assumption $(2.6)$ on $F$, with a constant $C=C(u)$, we find

$$
-\frac{d}{d t}\langle d E(u), w\rangle \leq C \int_{\mathbb{R}^{n}}\left(F(w)+w^{2}\right) d x
$$


If $m>0$, from (2.17), (2.20), we obtain

$$
\mathrm{I}(0)-\mathrm{I}(\mathrm{t}) \leq \mathrm{C} \int_{0}^{\mathrm{t}} \mathrm{E}(w(s)) \mathrm{ds}
$$

Moreover, estimating

$$
\begin{aligned}
\|w(\mathrm{t})\|_{\mathrm{L}^{2}}^{2} & \leq 2 \int_{0}^{\mathrm{t}} \int_{\mathbb{R}^{n}}\left|w_{\mathrm{t}}(\mathrm{s}) w(\mathrm{~s})\right| \mathrm{d} x \mathrm{~d} \mathrm{~s}+\|w(0)\|_{\mathrm{L}^{2}}^{2} \\
& \leq \int_{0}^{\mathrm{t}} \int_{\mathbb{R}^{n}}\left(|\mathrm{D} w(\mathrm{~s})|^{2}+|w(\mathrm{~s})|^{2}\right) \mathrm{d} x \mathrm{~d} \mathrm{~s}+\|w(0)\|_{\mathrm{L}^{2}}^{2},
\end{aligned}
$$

we have

$$
\|w(t)\|_{L^{2}}^{2} \leq C \int_{0}^{t} E(w(s)) d s+C E(w(0))
$$

In the case when $m=0$, we use Minkowski's inequality to bound

$$
\|w(t)\|_{L^{2}} \leq \int_{0}^{t}\left\|w_{t}(s)\right\|_{L^{2}} \mathrm{~d} s+\|w(0)\|_{L^{2}}
$$

Hölder's inequality then gives

$$
\|w(\mathrm{t})\|_{\mathrm{L}^{2}}^{2} \leq 2 \mathrm{~T} \int_{0}^{\mathrm{t}}\left\|w_{\mathrm{t}}(\mathrm{s})\right\|_{\mathrm{L}^{2}}^{2} \mathrm{ds}+2\|w(0)\|_{\mathrm{L}^{2}}^{2} \leq 4 \mathrm{~T} \int_{0}^{\mathrm{t}} \mathrm{E}(w(\mathrm{~s})) \mathrm{d} s+2\|w(0)\|_{\mathrm{L}^{2}}^{2}
$$

and from (2.20) we obtain that

$$
\mathrm{I}(0)-\mathrm{I}(\mathrm{t}) \leq \mathrm{C}\left(1+\mathrm{T}^{2}\right) \int_{0}^{\mathrm{t}} \mathrm{E}(w(\mathrm{~s})) \mathrm{ds}+\mathrm{CT}\|w(0)\|_{\mathrm{L}^{2}}^{2} .
$$

By (2.4) for the remaining term in the expansion (2.14), we have

$$
\begin{aligned}
\mathrm{II}(\mathrm{t}) & \geq \int_{\{\mathrm{t}\} \times \mathbb{R}^{n}}\left(\frac{|\mathrm{D} w|^{2}+m w^{2}}{2}+\varepsilon \mathrm{F}(w)-C w^{2}\right) d x \\
& \geq \varepsilon \mathrm{E}(w(\mathrm{t}))-\mathrm{C}\|w(\mathrm{t})\|_{\mathrm{L}^{2}}^{2},
\end{aligned}
$$

while at time $t=0$ by $(2.5)$ we have

$$
\begin{aligned}
\mathrm{II}(0) & \leq \int_{\{0\} \times \mathbb{R}^{n}}\left(\frac{|\mathrm{D} w|^{2}+m w^{2}}{2}+\mathrm{CF}(w)+C w^{2}\right) d x \\
& \leq \mathrm{CE}(w(0))+\mathrm{C}\|w(0)\|_{\mathrm{L}^{2}}^{2},
\end{aligned}
$$

and the latter is bounded by $\mathrm{E}(w(0))$ if $m>0$. 
From (2.7), (2.9), and (2.14) now we obtain

$$
0 \leq \mathrm{E}(v(0))-\mathrm{E}(v(\mathrm{t}))=\mathrm{I}(0)-\mathrm{I}(\mathrm{t})+\mathrm{II}(0)-\mathrm{II}(\mathrm{t}) .
$$

If $m=0$, in view of (2.26) and (2.28), we find

$$
\begin{aligned}
\mathrm{II}(\mathrm{t}) & \leq \mathrm{I}(0)-\mathrm{I}(\mathrm{t})+\mathrm{II}(0) \\
& \leq \mathrm{C}\left(1+\mathrm{T}^{2}\right) \int_{0}^{\mathrm{t}} \mathrm{E}(w(\mathrm{~s})) \mathrm{d} s+\mathrm{CE}(w(0))+\mathrm{C}(1+\mathrm{T})\|w(0)\|_{\mathrm{L}^{2}}^{2}
\end{aligned}
$$

and with (2.25), (2.27) we obtain

$$
\begin{aligned}
\mathrm{E}(w(\mathrm{t}))+\|w(\mathrm{t})\|_{\mathrm{L}^{2}}^{2} & \leq \mathrm{CII}(\mathrm{t})+\mathrm{C}\|w(\mathrm{t})\|_{\mathrm{L}^{2}}^{2} \\
& \leq \mathrm{C}\left(1+\mathrm{T}^{2}\right) \int_{0}^{\mathrm{t}} \mathrm{E}(w(\mathrm{~s})) \mathrm{d} s+\mathrm{CE}(w(0))+\mathrm{C}(1+\mathrm{T})\|w(0)\|_{\mathrm{L}^{2}}^{2} .
\end{aligned}
$$

On the other hand, if $m>0$, we may use (2.27), followed by (2.29) and then (2.28) together with (2.21) and (2.23) to conclude

$$
\mathrm{E}(w(\mathrm{t})) \leq \mathrm{CII}(\mathrm{t})+\mathrm{C}\|w(\mathrm{t})\|_{\mathrm{L}^{2}}^{2} \leq \mathrm{C} \int_{0}^{\mathrm{t}} \mathrm{E}(w(\mathrm{~s})) \mathrm{d} s+\mathrm{CE}(w(0)) .
$$

The desired estimate follows from Gronwall's inequality.

Note that in the preceding proof assumption (2.5) is only used to bound the terms $\mathrm{I}(0), \mathrm{II}(0)$ which vanish in the case of Theorem 2.1.

As already remarked in [10], Theorem 2.1 is similar to the uniqueness result of Ladyzenskaya for the Navier-Stokes equations [4]. Moreover, Theorems 2.1 and 2.2 are related to results of Dafermos [1] and DiPerna [3] for hyperbolic systems of conservation laws; see also Dafermos [2, Chapter 5.3]. Note, however, that in contrast to [2] we do not require the energy to be nonincreasing in forward time. Thus, as we will presently explain, Theorem 2.1 also can be used to establish convergence in the energy norm of standard approximation schemes for (2.1), which in general only yield energy-class solutions.

For functions $f_{k}$ with primitive $F_{k}(s)=\int_{0}^{s} f_{k}(r) d r, k \in \mathbb{N}$, consider solutions $u_{k}$ to the Cauchy problem

$$
u_{k, t t}-\Delta u_{k}+m u_{k}+f_{k}\left(u_{k}\right)=0 \quad \text { on } \mathbb{R} \times \mathbb{R}^{n}
$$

with Cauchy data

$$
\left(\mathfrak{u}_{k}, \mathfrak{u}_{k, t}\right)_{\left.\right|_{t=0}}=\left(\mathfrak{u}_{k 0}, u_{k 1}\right) \in H^{1} \times L^{2}\left(\mathbb{R}^{n}\right) .
$$


Theorem 2.3. Suppose (2.1) admits a smooth solution $u \in C^{\infty}\left(\mathbb{R} \times \mathbb{R}^{n}\right)$ for given Cauchy data $\left(u, u_{t}\right)_{\left.\right|_{t=0}}=\left(u_{0}, u_{1}\right) \in C_{0}^{\infty} \times C_{0}^{\infty}\left(\mathbb{R}^{n}\right)$, and let $\left(u_{k}\right)$ be a family of energy class solutions to (2.33), (2.34), where $f$ satisfies (2.3)-(2.6) and where the functions $f_{k}$ satisfy condition (2.3) with a uniform constant $C$ for all $k \in \mathbb{N}$. Also suppose that as $k \rightarrow \infty$ there holds $f_{k} \rightarrow$ f locally uniformly, while

$$
\left(u_{k 0}, u_{k 1}, F_{k}\left(u_{k 0}\right)\right) \longrightarrow\left(u_{0}, u_{1}, F\left(u_{0}\right)\right) \text { in } H^{1} \times L^{2} \times L^{1}\left(\mathbb{R}^{n}\right)
$$

Then there is strong convergence in the energy norm

$$
\left(u_{k}, u_{k, t}, F_{k}\left(u_{k}\right)\right) \longrightarrow\left(u, u_{t}, F(u)\right) \quad \text { in } L_{\text {loc }}^{\infty}\left(\mathbb{R} ; H^{1} \times L^{2} \times L^{1}\left(\mathbb{R}^{n}\right)\right)
$$

Proof of Theorem 2.3. In view of the energy inequality (2.8), as $k \rightarrow \infty$, we have

$$
E_{k}\left(u_{k}(t)\right)=\int_{\{t\} \times \mathbb{R}^{n}}\left(\frac{\left|D u_{k}+m u_{k}\right|^{2}}{2}+F_{k}\left(u_{k}\right)\right) d x \leq E_{k}\left(u_{k}(0)\right) \longrightarrow E(u(0)) .
$$

Hence, for a subsequence $\left(u_{k}\right)$, we have $D u_{k} \rightarrow$ Dv weakly- $*$ in $L^{\infty}\left(\mathbb{R} ; L^{2}\left(\mathbb{R}^{n}\right)\right)$ and $u_{k} \rightarrow v$ almost everywhere as $k \rightarrow \infty$; moreover, $\mathfrak{u}_{k}(0)=u_{k 0} \rightarrow v(0)=u(0)$ in $L^{2}\left(\mathbb{R}^{n}\right)$. In view of (2.3) and (2.37), it follows that $f_{k}\left(u_{k}\right) \rightarrow f(v)$ in $\left.L_{\text {loc }}^{1}\left(\mathbb{R} \times \mathbb{R}^{n}\right)\right)$. Passing to the limit in the weak form

$$
\int_{0}^{\infty} \int_{\mathbb{R}^{n}}\left(\mathfrak{u}_{k, t} \varphi_{t}-\nabla \mathfrak{u}_{k} \nabla \varphi-m u_{k} \varphi-f_{k}\left(u_{k}\right) \varphi\right) d x d t+\int_{\{0\} \times \mathbb{R}^{n}} u_{k 1} \varphi d x=0
$$

of (2.33) for any $\varphi \in C_{0}^{\infty}\left(\mathbb{R} \times \mathbb{R}^{n}\right)$, we then see that $v$ weakly solves $(2.1)$ with $v_{t}(0)=u_{1}$; that is, $v$ also satisfies (2.2). Finally, (2.37) together with weak lower semicontinuity on the one hand and Fatou's lemma on the other yields

$$
E(v(t)) \leq \liminf _{k \rightarrow \infty} E_{k}\left(u_{k}(t)\right) \leq \liminf _{k \rightarrow \infty} E_{k}\left(u_{k}(0)\right)=E(u(0))=E(v(0))
$$

for almost all $t$, and $v$ is an energy-class solution of (2.1), (2.2). From Theorem 2.1 then we conclude that $v=u$. The inequality (2.39) together with (2.7) now shows that $\mathrm{E}(\mathrm{u}(\mathrm{t}))=$ $\liminf _{k \rightarrow \infty} E_{k}\left(u_{k}(t)\right)$ and therefore that

$$
\|\mathrm{Du}(\mathrm{t})\|_{\mathrm{L}^{2}}=\liminf _{k \rightarrow \infty}\left\|\mathrm{D} u_{k}(t)\right\|_{L^{2}}, \quad \int_{\mathbb{R}^{n}} F(u(t)) d x=\liminf _{k \rightarrow \infty} \int_{\mathbb{R}^{n}} F_{k}\left(u_{k}(t)\right) d x
$$


for almost every $t \geq 0$. Hence, $D u_{k} \rightarrow D u$ in $L^{2}\left(\mathbb{R}^{n}\right), F_{k}\left(u_{k}\right) \rightarrow F(u)$ in $L^{1}\left(\mathbb{R}^{n}\right)$ strongly as $k \rightarrow \infty$ for almost all $t \geq 0$.

To see that the convergence is uniform, observe that $u$ is smooth by assumption with compact support, locally uniformly in time, and therefore also is bounded, locally uniformly in time. Since $F_{k}(s)$ by $(2.3)$ is nondecreasing in $s$ for $s>0$, at any point in space-time, we either have $\left|\mathfrak{u}_{k}\right| \geq|u|$ and

$$
F_{k}\left(u_{k}\right) \geq F_{k}(u) \geq F(u)-o(1)
$$

or $\left|u_{k}\right| \leq|u| \leq C=C(u, T)$ and

$$
\left|F_{k}\left(u_{k}\right)-F(u)\right| \leq\left|F\left(u_{k}\right)-F(u)\right|+o(1) \leq C\left|u_{k}-u\right|+o(1)
$$

with an error o $(1) \rightarrow 0$ locally uniformly in time as $k \rightarrow \infty$. It follows that

$$
\int_{\{t\} \times \mathbb{R}^{n}} F_{k}\left(u_{k}\right) d x \geq \int_{\{t\} \times \mathbb{R}^{n}} F(u) d x-o(1),
$$

and then that

$$
\left\|D u_{k}(t)\right\|_{L^{2}}^{2}-\|D u(t)\|_{L^{2}}^{2} \leq 2\left(E_{k}\left(u_{k}(t)\right)-E(u(t))\right)+o(1) \leq o(1),
$$

where o(1) $\rightarrow 0$ locally uniformly in time. Write

$$
\left\|\left(D u_{k}-D u\right)(t)\right\|_{L^{2}}^{2}=\left\|D u_{k}(t)\right\|_{L^{2}}^{2}-\|D u(t)\|_{L^{2}}^{2}-2 \int_{\{t\} \times \mathbb{R}^{n}}\left(D u_{k}-D u\right) \cdot D u d x
$$

and observe that with error o(1) $\rightarrow 0$ as $k \rightarrow \infty$, we have

$$
\int_{\{t\} \times \mathbb{R}^{n}}\left(D u_{k}-D u\right) \cdot D u d x=\int_{0}^{t} \int_{\mathbb{R}^{n}} \frac{d}{d t}\left(\left(D u_{k}-D u\right) \cdot D u\right) d x d s+o(1) .
$$

We may use (2.1) and (2.33) to shift all second derivatives occurring in the integral on the right to the smooth factor $u$. From weak space-time $\mathrm{L}^{2}$-convergence $\mathrm{D} u_{k} \rightarrow D u$, it then follows that

$$
\int_{\{t\} \times \mathbb{R}^{n}}\left(D u_{k}-D u\right) \cdot D u d x \longrightarrow 0, \quad \text { locally uniformly in } t
$$

which together with (2.44) yields strong convergence $D u_{k}(t) \rightarrow D u(t)$ in $L^{2}\left(\mathbb{R}^{n}\right)$ as $k \rightarrow$ $\infty$, locally uniformly in time. When combined with (2.37), (2.41), and (2.42), this fact then also gives locally uniform convergence $F_{k}\left(u_{k}(t)\right) \rightarrow F(u(t))$ in $L^{1}\left(\mathbb{R}^{n}\right)$. Since this argument applies to any subsequence, the assertion follows. 


\section{Nonlinear Schrödinger equations}

Consider now the Cauchy problem for the equation

$$
i u_{t}-\Delta u+m u+f(u)=0 \quad \text { on } \mathbb{R} \times \mathbb{R}^{n}
$$

with data

$$
u_{\mid t=0}=u_{0} \in C_{0}^{\infty}\left(\mathbb{R}^{n}\right) .
$$

Again we assume $m \geq 0$ and that $f$ satisfies conditions similar to the ones in Section 2, adapted to the complex setting. We suppose that $f$ is of the form $f(u)=u F^{\prime}\left(|u|^{2} / 2\right)$ for some smooth $\left(\mathrm{C}^{2}\right.$-) function $\mathrm{F}: \mathbb{R} \rightarrow \mathbb{R}$. The analogue of (2.3) then is the condition

$$
0 \leq s F^{\prime}(s) \leq \mathrm{CF}(\mathrm{s})
$$

for some constant $C$, while instead of conditions (2.4)-(2.6) we require that for all $R>0$ there exist numbers $\varepsilon=\varepsilon(R)>0, C=C(R)$ such that for all $w$ there hold

$$
\begin{aligned}
& F\left(\frac{|u+w|^{2}}{2}\right)-F\left(\frac{|u|^{2}}{2}\right)-f(u) \cdot w \geq \varepsilon F\left(\frac{|w|^{2}}{2}\right)-C|w|^{2}, \\
& \left|F\left(\frac{|u+w|^{2}}{2}\right)-F\left(\frac{|u|^{2}}{2}\right)-f(u) \cdot w\right|+|f(u+w)-f(u)-\langle d f(u), w\rangle| \\
& \quad+|f(u+w)-f(u)||w| \leq C\left(|w|^{2}+F\left(\frac{|w|^{2}}{2}\right)\right),
\end{aligned}
$$

provided $|u| \leq R$. Here and in the following for brevity we denote by

$$
\mathrm{a} \cdot \mathrm{b}=\operatorname{Re}(\mathrm{a} \overline{\mathrm{b}})
$$

the Hermitian inner product.

Similar to (2.7), the energy identity

$$
E(u(t))=\int_{\{t\} \times \mathbb{R}^{n}}\left(\frac{|\nabla u|^{2}+m u^{2}}{2}+F\left(\frac{|u|^{2}}{2}\right)\right) d x=E(u(0))
$$

will be valid for sufficiently regular solutions $u$ of (3.1), as can be seen by taking the Hermitian inner product of (3.1) with $u_{t}$ and integrating. Upon testing (3.1) with the function $i u$, we also find the conservation law

$$
\|\mathfrak{u}(\mathrm{t})\|_{\mathrm{L}^{2}}=\|\mathfrak{u}(0)\|_{\mathrm{L}^{2}}
$$


for the charge of system (3.1). Observe that in contrast to the case of (2.1), classical solutions to (3.1), (3.2) need no longer have spatially compact support. However, in many cases, it is possible to exhibit solutions that are bounded in any $\mathrm{H}^{\mathrm{s}}$-norm; see, for instance, Strauss [9]. These solutions together with their derivatives again will be uniformly bounded on any time-slice and will also decay sufficiently fast near spatial infinity for (3.7) and (3.8) to hold.

Just as in the case of the wave equation, for nonlinearities with "supercritical" growth, it is not known if the Cauchy problem (3.1), (3.2) will admit global smooth solutions. However, if we assume (3.3), standard approximation procedures yield the existence of weak solutions $u$ with $\nabla u, m u \in L^{\infty}\left(\mathbb{R} ; L^{2}\left(\mathbb{R}^{n}\right)\right)$ and such that $F(u) \in L^{\infty}\left(\mathbb{R} ; L^{1}\left(\mathbb{R}^{n}\right)\right)$, satisfying (3.1) in the sense of distributions and so that the energy inequality

$$
E(u(t)) \leq E(u(0))
$$

holds for all $t$; see Strauss [9, Theorem 3.1]. For these "energy-class" solutions again the $\operatorname{map} \mathrm{t} \mapsto \nabla \mathrm{u}(\mathrm{t}) \in \mathrm{L}^{2}\left(\mathbb{R}^{\mathrm{n}}\right)$ is weakly continuous and (3.9) ensures that the initial data (3.2) are assumed continuously in $\mathrm{H}^{1}$. Finally, also (3.8) holds for almost every time $t$.

We then have the following uniqueness result.

Theorem 3.1. Suppose $u \in \bigcap_{s \geq 0} L^{\infty}\left(\mathbb{R} ; H^{s}\left(\mathbb{R}^{n}\right)\right)$ solves (3.1) with Cauchy data $u_{\left.\right|_{t=0}}=$ $u_{0} \in C_{0}^{\infty}\left(\mathbb{R}^{n}\right)$, where $f$ satisfies (3.3)-(3.5). Also, let $v$ be an energy-class solution to (3.1), (3.2), satisfying the energy inequality (3.9). Then $u \equiv v$.

Again, Theorem 3.1 is contained in the following stability result.

Theorem 3.2. Suppose $u \in \bigcap_{s \geq 0} L^{\infty}\left(\mathbb{R} ; H^{s}\left(\mathbb{R}^{n}\right)\right)$ solves (3.1) with Cauchy data $u_{\mid t=0}=$ $u_{0} \in C_{0}^{\infty}\left(\mathbb{R}^{n}\right)$, where $f$ satisfies (3.3)-(3.5). For Cauchy data $v_{\left.\right|_{t=0}}=v_{0} \in H^{1}\left(\mathbb{R}^{n}\right)$ with $\mathrm{F}\left(v_{0}\right) \in \mathrm{L}^{1}\left(\mathbb{R}^{n}\right)$, let $v$ be an energy-class solution to (3.1), satisfying the energy inequality

$$
\mathrm{E}(v(\mathrm{t})) \leq \mathrm{E}(v(0)) \quad \forall \mathrm{t}
$$

Then, if $m>0$, letting $w=v-u$, for any time $t>0$ with uniform constants $C_{i}=C_{i}(u)$,

$$
E(w(t)) \leq C_{1} e^{C_{2} t} E(w(0)) .
$$

If $m=0$, for any time $T>0$ with a constant $C=C(u, T)$,

$$
\mathrm{E}(w(\mathrm{t}))+\|w(\mathrm{t})\|_{\mathrm{L}^{2}}^{2} \leq \mathrm{C}\left(\mathrm{E}(w(0))+\|w(0)\|_{\mathrm{L}^{2}}^{2}\right)
$$

uniformly for $0<\mathrm{t}<\mathrm{T}$. 
Proof. The proof is very similar to the case of the wave equation. Again we consider only forward time $t \geq 0$.

The function $w$ satisfies the equation

$$
i w_{t}-\Delta w+m w+f(u+w)-f(u)=0 \quad \text { on } \mathbb{R} \times \mathbb{R}^{n} .
$$

Expanding as in Section 2, for any $t>0$, we have

$$
E(v)=E(u)+I+I I,
$$

where

$$
\mathrm{I}=\langle\mathrm{dE}(\mathrm{u}), w\rangle=\int_{\mathbb{R}^{n}}(\nabla \mathrm{u} \cdot \nabla \mathfrak{w}+\mathrm{mu} \cdot \mathfrak{w}+\mathrm{f}(\mathfrak{u}) \cdot \mathfrak{w}) \mathrm{d} x
$$

and with

$$
\mathrm{II}=\int_{\mathbb{R}^{n}}\left(\frac{|\nabla w|^{2}+m w^{2}}{2}+\left(\mathrm{F}\left(\frac{|\mathfrak{u}+w|^{2}}{2}\right)-\mathrm{F}\left(\frac{|\mathrm{u}|^{2}}{2}\right)-\mathrm{f}(\mathrm{u}) \cdot w\right)\right) \mathrm{d} x .
$$

With $\eta_{k} \in C_{0}^{\infty}(] 0, t[)$ approximating the characteristic function of $[0, t]$ as before, again we find

$$
I(t)-I(0)=\lim _{k \rightarrow \infty} \int_{0}^{t} \eta_{k} \frac{d}{d t}\langle d E(u), w\rangle d s .
$$

To compute the time derivative, it is convenient to use the equation

$$
\begin{aligned}
\left.\frac{d}{d s}\right|_{s=0} & \frac{d}{d t} F\left(\frac{|u+s w|^{2}}{2}\right) \\
& =\left.\frac{d}{d s}\right|_{s=0}\left(f(u+s w) \cdot\left(u_{t}+s w_{t}\right)\right)=\langle d f(u), w\rangle \cdot u_{t}+f(u) \cdot w_{t} \\
& =\left.\frac{d}{d t} \frac{d}{d s}\right|_{s=0} F\left(\frac{|u+s w|^{2}}{2}\right)=\frac{d}{d t} f(u) \cdot w+f(u) \cdot w_{t},
\end{aligned}
$$

yielding that

$$
\frac{d}{d t} f(u) \cdot w=\langle d f(u), w\rangle \cdot u_{t} .
$$

Therefore, upon integrating by parts, we have

$$
\begin{aligned}
& \frac{\mathrm{d}}{\mathrm{dt}}\langle\mathrm{dE}(\mathrm{u}), w\rangle \\
& \quad=\int_{\mathbb{R}^{n}} \frac{\mathrm{d}}{\mathrm{dt}}(\nabla \mathrm{u} \cdot \nabla w+\mathrm{mu} \cdot w+\mathrm{f}(\mathrm{u}) \cdot w) \mathrm{d} x \\
& \quad=\int_{\mathbb{R}^{n}}\left((-\Delta \mathrm{u}+\mathrm{mu}+\mathrm{f}(\mathrm{u})) \cdot w_{\mathrm{t}}+(-\Delta w+\mathrm{m} w+\langle\mathrm{df}(\mathrm{u}), w\rangle) \cdot \mathrm{u}_{\mathrm{t}}\right) \mathrm{d} x
\end{aligned}
$$


which is well defined as a distribution on ]0,t[. Using (3.1) and (3.13), respectively, and observing that $\left(\mathfrak{i} u_{t}\right) \cdot w_{t}+\left(\mathfrak{i} w_{t}\right) \cdot u_{t}=0$, in view of assumption (3.5) we then obtain

$$
\begin{aligned}
-\frac{d}{d t}\langle d E(u), w\rangle & =\int_{\mathbb{R}^{n}}(f(u+w)-f(u)-\langle d f(u), w\rangle) \cdot u_{t} d x \\
& \leq C \int_{\mathbb{R}^{n}}\left(|w|^{2}+F\left(\frac{|w|^{2}}{2}\right)\right) d x .
\end{aligned}
$$

We first consider the case when $m>0$. In this case, the $\mathrm{L}^{2}$-norm of $w$ is bounded by the energy and upon inserting (3.21) into (3.17) we obtain

$$
\mathrm{I}(0)-\mathrm{I}(\mathrm{t}) \leq \mathrm{C} \int_{0}^{\mathrm{t}} \mathrm{E}(w(\mathrm{~s})) \mathrm{ds}
$$

As in Section 2, by using the assumptions (3.7), (3.9) together with the expansion (3.14), we obtain

$$
0 \leq \mathrm{E}(v(0))-\mathrm{E}((v(\mathrm{t}))=\mathrm{I}(0)-\mathrm{I}(\mathrm{t})+\mathrm{II}(0)-\mathrm{II}(\mathrm{t})
$$

By (3.4), similar to (2.27), we have the lower bound

$$
\mathrm{II}(\mathrm{t}) \geq \int_{\{\mathrm{t}\} \times \mathbb{R}^{n}}\left(\frac{|\nabla w|^{2}+m w^{2}}{2}+\varepsilon \mathrm{F}(w)-C w^{2}\right) d x \geq \varepsilon \mathrm{E}(w(\mathrm{t}))-\mathrm{C}\|w(\mathrm{t})\|_{\mathrm{L}^{2}}^{2},
$$

while (3.5) allows to estimate

$$
\mathrm{II}(0) \leq \int_{\{0\} \times \mathbb{R}^{n}}\left(\frac{|\nabla w|^{2}+m w^{2}}{2}+\mathrm{CF}(w)+C w^{2}\right) \mathrm{d} x \leq \mathrm{CE}(w(0))+\mathrm{C}\|w(0)\|_{\mathrm{L}^{2}}^{2},
$$

and the latter again is bounded by $\mathrm{E}(w(0))$ if $\mathrm{m}>0$. In order to conclude, however, we still need to derive a bound for the $\mathrm{L}^{2}$-norm of $w$. Observe that the argument from Section 2 cannot be used in the case of the Schrödinger equation since the time derivative is not part of the energy. 
Fortunately, the bound (3.9) suffices to justify testing equation (3.13) with the function iw. Also using (3.5), in the distribution sense, we can bound

$$
\frac{\mathrm{d}}{\mathrm{dt}}\|w\|_{\mathrm{L}^{2}}^{2}=2 \int_{\mathbb{R}^{n}}(\mathrm{f}(\mathfrak{u})-\mathrm{f}(\mathrm{u}+\mathfrak{w})) \cdot(\mathfrak{i} w) \mathrm{d} x \leq \mathrm{C}_{1} \int_{\mathbb{R}^{n}}\left(|\mathfrak{w}|^{2}+\mathrm{F}\left(\frac{|w|^{2}}{2}\right)\right) \mathrm{d} x
$$

with a constant $C_{1}=C_{1}(u)>0$. If $m>0$, we may further estimate the last term in (3.26) by the energy to find

$$
\|w(t)\|_{L^{2}}^{2} \leq\|w(0)\|_{L^{2}}^{2}+C \int_{0}^{t} E(w(s)) d s \leq C E(w(0))+C \int_{0}^{t} E(w(s)) d s
$$

similar to (2.23). Inserting the bounds (3.22), (3.24), (3.25), and (3.27) into (3.23), we obtain

$$
E(w(t)) \leq C I I(t)+C\|w(t)\|_{L^{2}}^{2} \leq C E(w(0))+C \int_{0}^{t} E(w(s)) d s
$$

in this case and we conclude from Gronwall's inequality.

On the other hand, if $m=0$, from (3.26), we have

$$
\|w(t)\|_{L^{2}}^{2} \leq e^{C_{1} t}\|w(0)\|_{L^{2}}^{2}+C_{1} \int_{0}^{t} e^{C_{1}(t-s)} E(w(s)) d s
$$

yielding the bound

$$
\mathrm{I}(0)-\mathrm{I}(\mathrm{t}) \leq \mathrm{C}\|w(0)\|_{\mathrm{L}^{2}}^{2}+\mathrm{C} \int_{0}^{\mathrm{t}} \mathrm{E}(w(\mathrm{~s})) \mathrm{ds}
$$

instead of (3.22) with constants $C=C(u, T)$. Inserting the bound (3.30) into (3.23) and using (3.29) together with (3.24), (3.25), we then also find

$$
\begin{aligned}
\mathrm{E}(w(\mathrm{t}))+\|w(\mathrm{t})\|_{\mathrm{L}^{2}}^{2} & \leq \mathrm{CII}(\mathrm{t})+\mathrm{C}\|w(\mathrm{t})\|_{\mathrm{L}^{2}}^{2} \\
& \leq \mathrm{C}\left(\mathrm{E}(w(0))+\|w(0)\|_{\mathrm{L}^{2}}^{2}\right)+\mathrm{C} \int_{0}^{\mathrm{t}} \mathrm{E}(w(\mathrm{~s})) \mathrm{ds}
\end{aligned}
$$

with constants $\mathrm{C}=\mathrm{C}(\mathrm{u}, \mathrm{T})$, as claimed. 


\section{Acknowledgments}

I would like to thank Walter Strauss and Constantine Dafermos for very stimulating comments and, in particular, for suggesting to investigate the approach in [10] also in the context of Schrödinger equations and with regard to the issue of stability. This note was written in September 2005 at the Mittag-Leffler Institute whose hospitality and support I gratefully acknowledge.

\section{References}

[1] C. M. Dafermos, The second law of thermodynamics and stability, Archive for Rational Mechanics and Analysis 70 (1979), no. 2, 167-179.

[2] , Hyperbolic Conservation Laws in Continuum Physics, 2nd ed., Grundlehren der mathematischen Wissenschaften, vol. 325, Springer, Berlin, 2005.

[3] R. J. DiPerna, Uniqueness of solutions to hyperbolic conservation laws, Indiana University Mathematics Journal 28 (1979), no. 1, 137-188.

[4] O. A. Ladyzhenskaya, The Mathematical Theory of Viscous Incompressible Flow, 2nd ed., Mathematics and Its Applications, vol. 2, Gordon and Breach Science, New York, 1969.

[5] J.-L. Lions, Une remarque sur les problèmes d'évolution non linéaires dans des domaines non cylindriques, Revue Roumaine de Mathématiques Pures et Appliquées 9 (1964), 11-18.

[6] I. E. Segal, The global Cauchy problem for a relativistic scalar field with power interaction, Bulletin de la Société Mathématique de France 91 (1963), 129-135.

[7] J. Shatah and M. Struwe, Geometric Wave Equations, 2nd ed., Courant Lecture Notes in Mathematics, vol. 2, Courant Institute of Mathematical Sciences, New York; American Mathematical Society, Rhode Island, 2000.

[8] W. A. Strauss, On weak solutions of semi-linear hyperbolic equations, Anais da Academia Brasileira de Ciências 42 (1970), 645-651.

[9] Nonlinear Wave Equations, CBMS Regional Conference Series in Mathematics, vol. 73, American Mathematical Society, Rhode Island, 1989.

[10] M. Struwe, Uniqueness for critical nonlinear wave equations and wave maps via the energy inequality, Communications on Pure and Applied Mathematics 52 (1999), no. 9, 1179-1188.

Michael Struwe: Departement Mathematik, ETH Zürich, 8092 Zürich, Switzerland

E-mail address: struwe@math.ethz.ch 